\title{
NeuroHeal Improves Muscle Regeneration after Injury
}

\author{
Sara Marmolejo-Martínez-Artesero ${ }^{1}{ }^{(\mathbb{D}}$, David Romeo-Guitart $1,2, * \mathbb{D}$, Vanesa Venegas ${ }^{3,4}$, Mario Marotta ${ }^{3,4}$ and \\ Caty Casas ${ }^{1,+}$ (iD
}

1 Department of Cell Biology, Physiology and Immunology, Institut de Neurociències (INc), Universitat Autònoma de Barcelona (UAB), Bellaterra, 08193 Barcelona, Spain; Sara.Marmolejo@uab.cat

2 Laboratory "Hormonal regulation of brain development and functions"-Team 8, Institut Necker Enfants-Malades (INEM), INSERM U1151, Université Paris Descartes, Sorbonne Paris Cité, 75015 Paris, France

3 Leitat Technological Center, Carrer de la Innovació 2, Terrassa, 08225 Barcelona, Spain; vanesa.venegas@vhir.org (V.V.); mmarotta@leitat.org (M.M.)

4 Bioengineering, Cell Therapy and Surgery in Congenital Malformations Laboratory, Vall d'Hebron Institut de Recerca (VHIR), Universitat Autònoma de Barcelona (UAB), 08035 Barcelona, Spain

* Correspondence: david.romeo-guitart@inserm.fr; Tel.: +33-0140-615-357

$+\quad$ Died 29 June 2020.

check for updates

\section{Citation:}

Marmolejo-Martínez-Artesero, S.; Romeo-Guitart, D.; Venegas, V.; Marotta, M.; Casas, C. NeuroHeal Improves Muscle Regeneration after Injury. Cells 2021, 10, 22. https://dx.doi.org/10.3390/ cells10010022

\section{Received: 6 December 2020}

Accepted: 22 December 2020

Published: 24 December 2020

Publisher's Note: MDPI stays neutral with regard to jurisdictional claims in published maps and institutional affiliations.

Copyright: () 2020 by the authors. Licensee MDPI, Basel, Switzerland. This article is an open access article distributed under the terms and conditions of the Creative Commons Attribution (CC BY) license (https: / / creativecommons.org/ licenses/by/4.0/).

\begin{abstract}
Musculoskeletal injuries represent a challenging medical problem. Although the skeletal muscle is able to regenerate and recover after injury, the process engaged with conservative therapy can be inefficient, leading to a high re-injury rate. In addition, the formation of scar tissue implies an alteration of mechanical properties in muscle. There is still a need for new treatments of the injured muscle. NeuroHeal may be one option. Published studies demonstrated that it reduces muscle atrophy due to denervation and disuse. The main objective of the present work was to assess the potential of NeuroHeal to improve muscle regeneration after traumatic injury. Secondary objectives included characterizing the effect of NeuroHeal treatment on satellite cell biology. We used a rat model of sport-induced injury in the gastrocnemius and analyzed the effects of NeuroHeal on functional recovery by means of electrophysiology and tetanic force analysis. These studies were accompanied by immunohistochemistry of the injured muscle to analyze fibrosis, satellite cell state, and fiber type. In addition, we used an in vitro model to determine the effect of NeuroHeal on myoblast biology and partially decipher its mechanism of action. The results showed that NeuroHeal treatment advanced muscle fiber recovery after injury in a preclinical model of muscle injury, and significantly reduced the formation of scar tissue. In vitro, we observed that NeuroHeal accelerated the formation of myotubes. The results pave the way for novel therapeutic avenues for muscle/tendinous disorders.
\end{abstract}

Keywords: muscle regeneration; NeuroHeal; satellite cells; Sirtuin 1; sport injury

\section{Introduction}

Musculoskeletal injuries represent a challenging problem, being the most common cause of severe long-term injuries, accounting for 10 to $55 \%$ of all sports-related injuries [1]. Muscle injuries are classified by cause of trauma and grade: an acute lesion caused by a direct trauma, such as muscle lacerations, an indirect trauma, such as strains, or by chronic disease or degenerative diseases, such as muscular dystrophies [2]. To repair this injury, skeletal muscle has regenerative capabilities to self-repair after trauma or in response to muscular dystrophies. This muscle reparative mechanism is divided into different phases: degeneration, inflammation, regeneration, remodeling, and innervation [3]. The regenerative process is mainly mediated by a specific type of stem cell, the satellite cell (SC) $[4,5]$, which, after being activated, follows a process of differentiation giving a newly formed or recovered myofiber [6]. Recent reports have provided evidence that SCs are essential for the regenerative process, which is directly dependent on the microenvironmental niche $[7,8]$. 
Current therapies for most muscle injuries are mainly directed to decrease pain and inflammation by NSAIDs drugs, supplemented with immobilization and external physical stimulation. In severe cases, surgery is required to restore the anatomic continuity and function. In some cases, the endogenous process of muscle repair proves insufficient, leading to loss of contractile tissue, fatty degeneration, and fibrotic scar tissue, which can cause long-term deficits in muscle structure and strength. Although in those cases medical need is unsolved, some promising therapies are under study. The use of molecular therapies, such as growth factors or platelet-rich-plasma (PRP), which releases growth factors, structural proteins, and interleukins and chemokines, try to foster a beneficial microenvironment to boost regenerative biological processes [3]. Although there is controversy regarding the clinical effects of PRP in humans [9], it was shown to promote beneficial effects on muscle recovery in a rat injury model [10]. Moreover, cell therapy is a promising approach to treat skeletal muscle injuries in pre-clinical settings [11], but not for all types of injuries. Therefore, all current available pharmacological and biological agents only relieve clinical symptoms and have limited or no effect on the progression of the underlining muscle disease.

In this study, we evaluated the therapeutic potential of NeuroHeal, a combination of two repurposed drugs (Acamprosate plus Ribavirin), previously designed using artificial intelligence [12,13]. Drug repurposing for other indications facilitates its readiness for clinical use, since their PD and PK are well-known in humans. We have previously reported its neuroprotective effects in different neurodegenerative models [12,14-16]. In addition, we recently observed that NeuroHeal protects the muscle by reducing atrophy induced by denervation or by immobilization [17], suggesting it may have also protective effects in other types of myopathies. NeuroHeal neuroprotective and neurodegenerative effects involve SIRT1 activation, and autophagy modulation $[14,15,18]$. Moreover, we have recently described that boosting SIRT1 activity is essential in the mechanism of action of NeuroHeal to reduce muscle atrophy [17]. Herein, we aimed to elucidate whether NeuroHeal has an effect on muscle regeneration by using an in vivo model of surgicallyinduced lesion which mimics the most frequent skeletal muscle lesions observed in human sports clinics. We also provide insight into the possible mechanism involved in the proregenerative effect of NeuroHeal.

\section{Materials and Methods}

\subsection{Muscle Injury Model}

All the experimental procedures were performed in accordance with Spanish (Real Decreto 53/2013) and European (2010/63/UE) legislation, approved by the Departament d'Agricultura, Ramaderia, Pesca, Alimentació i Medi Natural of the Catalan Government (Generalitat de Catalunya) and followed the ethical standards in sport and exercise science research $[10,19,20]$. The work was performed under the protocol number 2017/52.17 approved by the Institutional Animal Care and Use Committee at Vall d'Hebron Hospital Research Institute (Barcelona, Spain). Adult male Wistar rats 8 weeks of age (Harlan Laboratories) were used for the in vivo studies. Rats were anesthetized by an intraperitoneal (i.p.) injection of a mixture of ketamine (90 mg/kg, Ketaset) and xylazine (10 mg/kg, Rompun). The surgical procedure was performed as previously described $[10,20]$, inserting an $18 \mathrm{G}$ biopsy needle in the right medial gastrocnemius (GA) muscle at $3 \mathrm{~mm}$ from the muscletendon junction. Post-surgical analgesia $(0.01 \mathrm{mg} / \mathrm{kg}$ buprenorphine) was administered to all operated animals for $24 \mathrm{~h}$ after surgically-induced skeletal muscle injury.

\subsection{Drug Treatment}

NeuroHeal mixture is composed of Acamprosate and Ribavirin. For in vivo experiments, we grounded Acamprosate (Merck, Darmstadt, Germany) and Ribavirin (Normon, Madrid, Spain) pills into a fine powder and daily administered $40 \mathrm{mg} / \mathrm{kg}$ and $26 \mathrm{mg} / \mathrm{kg}$, respectively, by gavage, dissolved in $1 \mathrm{~mL}$ of water at a concentration of $2.2 \mathrm{mM}$ Acamprosate and $1 \mathrm{mM}$ Ribavirin. To test whether NeuroHeal may accelerate muscle regeneration after 
damage, we used a model of injured GA muscle, comparing a group of rats treated with NeuroHeal $(n=5)$ and a group of rats receiving vehicle $(n=5)$, by gavage administration.

\subsection{Electrophysiology}

Rats were anesthetized by i.p. administration of ketamine and xylazine, and kept warm with a thermostated blanket. The sciatic nerve was stimulated with single pulses of increasing intensity by means of transcutaneous electrodes placed at the sciatic notch. The compound muscle action potential (CMAP) of the GA muscle was recorded to measure the maximum CMAP amplitude. The tests were performed using an electromyography (EMG) apparatus (Synergy Medelec, Viasys HealthCare). The CMAP amplitude from baseline to peak was used as a readout of the GA muscle integrity. The percentage of CMAP recovery was calculated as the ratio of injured/uninjured contralateral values. The nerve conduction test was performed before surgical injury and at 3, 7, and 14 days post-injury (dpi).

\subsection{Measurement of Muscle Force}

To measure contractile muscle force, rats were anesthetized by i.op. injection of ketamine and xylazine. Animals were placed in a prone position and the limbs were immobilized. The Achilles tendon was separated from the calcaneus and attached to a force transducer (Wide Range Force Transducer, MLT 1030/D; ADInstruments, Sydney, Australia) connected to a PowerLab/16SP data acquisition hardware (ADInstruments). The sciatic nerve was exposed by lateral incision and an electrode placed around the nerve and connected in turn to a stimulator (Stimulus Isolator, FE180; ADInstruments). The GA muscles were covered with mineral oil (Sigma-Aldrich, Saint Louis, MO, USA) to prevent drying, and the room temperature was set at $25^{\circ} \mathrm{C}$. Muscle force was measured for right (injured) and left (control) GA muscles of each rat. Repeated isometric muscle twitches were induced at a frequency of $1 \mathrm{~Hz}$ (5 pulses) and a voltage of $5 \mathrm{~V}$. The twitch response was analyzed by contraction time (CT) and half-relaxation time (HRT). Following twitch stimulation or peak force (PF), maximum tetanus force (TetF) was induced by a train of stimuli with a frequency of $100 \mathrm{~Hz}$, a pulse width of $0.1 \mathrm{~ms}$ and a voltage of $5 \mathrm{~V}$. The percentage of the TetF was calculated with the percentage of the ipsi versus the contra GA of each animal. Animals were euthanized by anesthetic overdose immediately after finishing muscle force measurements and muscle samples were excised and processed for further histological analysis.

\subsection{Histology}

For histology studies, animals were euthanized by i.p. injection of an anesthetic overdose. The GA muscles were carefully excised, weighed, and immediately frozen in 2-methylbutane (Alfa Aesar, Haverhill, MA, USA), which was previously supercooled in liquid nitrogen, and were stored at $-80^{\circ} \mathrm{C}$ until further analysis.

To locate the area of injury, $10 \mu \mathrm{m}$ serial cross sections were made from the myotendinous junction. Muscle samples were embedded in Tissue-tek, sectioned in a cryotome (Leica, Heidelberg, Germany), and preserved at $-20^{\circ} \mathrm{C}$ until analysis. Hematoxylin and Eosin (H\&E) staining was performed in each series to cover $3 \mathrm{~mm}$ of the muscle to localize the injury. Each series was formed by five slices, having discarded $50 \mu \mathrm{m}$ between them. The samples to be compared were processed together the same day within the same slide, and image analysis for all groups was also performed the same day using the same microscope setting.

For the H\&E, nuclei were stained with Harris hematoxylin for 6 min followed by differentiation with an acid solution of $0.01 \% \mathrm{HCl}$ in ethanol. The cytoplasm was stained with eosin for $1 \mathrm{~min}$. Sections were dehydrated by graded ethanol $(50 \%, 70 \%, 96 \%$, and $100 \%$, and xylene twice, $5 \mathrm{~min}$ in each solution) and mounted with DPX mounting solution.

For immunofluorescence labeling, the slices were pre-treated with frozen acetone, to fix the samples, and with $10 \mathrm{mg} / \mathrm{mL} \mathrm{NaBH}$, to reduce autofluorescence for $80 \mathrm{~min}$ at $4{ }^{\circ} \mathrm{C}$. After washing with standard Phosphate Buffered Saline (PBS), the tissue was incubated in 
blocking solution ( $0.3 \%$ Triton-X-100 and $10 \%$ fetal bovine serum in PBS) for $1 \mathrm{~h}$ at room temperature. Samples were then incubated at $4{ }^{\circ} \mathrm{C}$ with primary antibodies containing solution (PBS with $0.15 \%$ triton $\mathrm{X}-100,5 \%$ FBS) overnight. The antibodies used were mouse anti-collagen type 1 (1:100, DBSH, Iowa City, IA, USA), mouse anti-developmental myosin heavy chain (dMyHC. 1:100, DSHB), mouse anti-fast myosin heavy chain (fMyHC. 1:100, DSHB), mouse anti-laminin (1:100, DSHB), mouse anti-MyoD (1:150; Santa Cruz, Dallas, TX, USA), mouse anti-Myogenin (1:100; Abcam, Cambridge, UK), rabbit anti-parvalbumin (1:100, Swant, Marly, Switzerland), mouse anti-Pax7 (1:100; DSHB), and mouse anti- $\alpha \beta$ slow myosin heavy chain (sMyHC. 1:100, DSHB). After several washes with $0.3 \%$ Triton-X100 in PBS, we added Alexa Fluor 488 and 594 conjugated secondary antibodies against the primary antibody (1:100; Jackson Immunoresearch, Cambridge, UK) and incubated for $1 \mathrm{~h}$ at room temperature. Counter-staining was performed with DAPI (Sigma) and mounted with Fluoromont (SouthernBiotech, Birmingham, UK) mounting solution. Images from different groups were taken under the same exposure time, sensibility, resolution, and microscope for each analyzed marker. Images were taken using a Nikon Eclipse Ni-E microscope equipped with a digital camera (Nikon DS-RiE, Nikon, Tokyo, Japan) and Nikon NIS-Element BR software (version 5.11.03, Nikon, Tokyo, Japan). Co-labeled fibers were determined as positive using a pseudocolor display by Image J software (version 1.46; National Institutes of Health), using images at $4 \times$ (fiber type markers) and $10 \times$ (myogenesis markers). For signal intensity analysis, randomly selected images were selected at $10 \times$ (collagen I) and $20 \times$ (parvalbumin), were transformed to grayscale, and after defining a threshold for background correction, immunoreactivity was analyzed by calculating the integrated density of a region of interest (ROI) and the total area of the image. For collagen I, the ROI was $1.49 \mathrm{~mm}^{2}$ and the ROI of parvalbumin was myofiber area for a total of 20 myofibers.

\subsection{Myoblast Differentiation and Analysis}

C2C12 myoblast cell line was grown in a medium composed of modified Eagle's medium high-glucose (DMEM, Life Technologies, Carlsbad, CA, USA) supplemented with $10 \%$ fetal bovine serum (Sigma-Aldrich), and 1\% penicillin/streptomycin solution (SigmaAldrich). Cells were kept in a humidified incubator at $37{ }^{\circ} \mathrm{C}$ under $5 \% \mathrm{CO}_{2}$. To initiate the experiments, cells were seeded at a density of $8.5 \times 10^{3}$ cell $/ \mathrm{mL}$, and after $24 \mathrm{~h}$ of culture and $80 \%$ of confluency, the medium was changed to a differentiation medium (DMEM supplemented with $2 \%$ horse serum (Sigma-Aldrich) with $1 \%$ penicillin/streptomycin solution), with or without NeuroHeal (55 $\mu \mathrm{M}$ acamprosate and $1 \mu \mathrm{M}$ ribavirin) and Ex527 (Sigma-Aldrich) at $10 \mu \mathrm{M}$, which was changed every 2 days, until the end of the experiment. At 0 (before changing to differentiation medium), 1, 3, and 5 div (days of in vitro) cells were fixed using $4 \%$ formaldehyde for $30 \mathrm{~min}$ and then washed out with TBS. For immunofluorescence labeling, cells were incubated in a blocking solution $(0.3 \%$ Triton-X-100 and 10\% normal donkey serum in TBS) for $1 \mathrm{~h}$ at room temperature. Samples were then incubated for $4 \mathrm{~h}$ at room temperature with primary antibodies containing solution (TBS with $0.3 \%$ triton X-100, 5\% FBS). The antibodies used were rabbit anti-ki67 (1:100; Abcam), mouse anti-MyoD (1:150; Santa Cruz Biotechnology), mouse anti-Myosin heavy chain (all fast isoforms) (MyHC, 1:20, DSHB), rabbit anti-parvalbumin (1:100, Swant), and mouse anti-Pax7 (1:100; DSHB). After several washes in TBS-0.1\% tween-20, we added Cy2 and Cy3-labeled secondary antibodies (1:200; Jackson Immunoresearch) were added and incubated for $50 \mathrm{~min}$ at room temperature. Counter-staining was performed with DAPI (Sigma) and mounting with Mowiol mounting medium (Southern Biotech). Images of $\mathrm{C} 2 \mathrm{C} 12$ cells from different groups were taken at $20 \times$ under the same exposure time, sensibility, and resolution for each marker analyzed with the same microscope system as above. Co-labeled fibers were determined as positive using a pseudocolor display by Image J software. For the fusion coefficient, a mature myotube was considered having three or more MyHC positive nuclei. For signal intensity analysis, images were transformed to grayscale and analyzed immunoreactivity by calculating the integrated density of a 
ROI, after defining a threshold for background correction. For MyoD, the ROI used was DAPI to analyze nuclei area and for parvalbumin, the ROI used was $5615.103 \mu \mathrm{m}^{2}$ for 15-20 myotubes for each condition.

\subsection{Statistical Analysis}

We performed an unpaired Student's $t$-test to compare two groups, a one-way analysis of variance (ANOVA) to compare three or more groups, and a two-way ANOVA to compare grouped data followed by Tukey's multiple comparison test, as appropriate. Data are presented as means \pm standard error of the mean (SEM) and differences were assumed to be significant for $p \leq 0.05$. Statistical analyses were conducted using GraphPad Prism 8 software.

\section{Results}

\subsection{Text}

\subsubsection{NeuroHeal Accelerates Muscle Fiber Function Recovery}

The lesion in the vehicle group caused a reduction of the CMAP amplitude in the GA muscle $(83.5 \% \pm 3.1 \%)$ compared to the contralateral non-injured muscle at 3 dpi (Figure 1B). Weekly follow-up of the injured animals showed a progressive recovery of the CMAP amplitude until normal values were reached at $14 \mathrm{dpi}$ in the NeuroHeal group, in comparison to the persistent reduction of the CMAP amplitude in the GA at $14 \mathrm{dpi}$ in the untreated group. These results evidenced that the treatment with NeuroHeal significantly improved muscle fiber recovery.

No significant differences were detected in GA muscle weight between the two groups and between ipsi- and contralateral GA muscle for each group at the end of follow-up (Figure S1). Regarding muscle force recovery, untreated animals showed an approximate $20 \%$ decrease in TetF of the injured versus contralateral leg $(79.1 \% \pm 2.3 \%)$, in comparison to NeuroHeal-treated group $(84.9 \% \pm 2.9 \%)$, without significant differences (Figure 1C). Moreover, the untreated group showed a shorter CT and HRT in comparison to the NeuroHeal-treated group (Figure 1D), coinciding with a PF non-significant increase in this group (Figure 1E). All these data suggested that NeuroHeal may be altering the muscle contractile properties regardless of injury.

\subsubsection{NeuroHeal Modulates Fiber Regeneration and Reduces Collagen Deposition}

To determine the effect of NeuroHeal of muscle repair, we analyzed the lesion area, previously identified by H\&E staining, with immunohistochemical labeling of markers of regenerating myofibers and of collagen deposition at $14 \mathrm{dpi}$. The number of total muscle fibers was not modified by the treatment (Figure 2 and Figure S2). Then, we evaluated the regeneration state of the injured fibers. We observed that NeuroHeal did not promote a change in the number of developmental MyHC (Figure 2 and Figure S2). In addition, we observed that NeuroHeal induced a non-significant increase in the number of fast MyHC fibers, and an increase of slow MyHC fibers (Figure 2 and Figure S2) in the injured GA muscle, at the lesion zone. On the other side, NeuroHeal treatment did not modify the distribution of fast and slow MyHC in the contralateral GA muscle (Figure S3). Finally, the NeuroHeal-treated group showed lower collagen I levels in comparison to the untreated group (Figure 2 and Figure S2). 
A

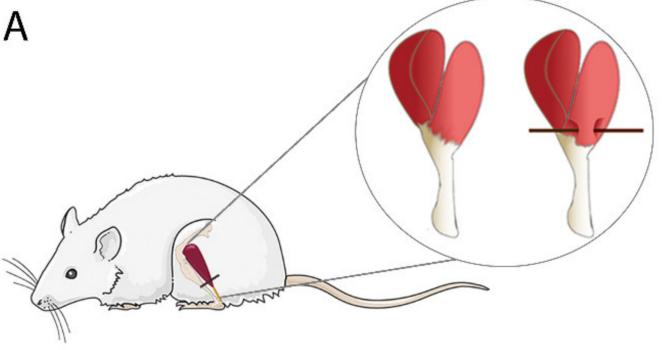

B

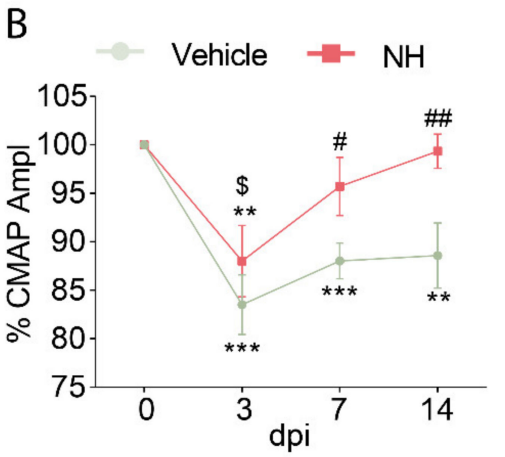

$\mathrm{D}$

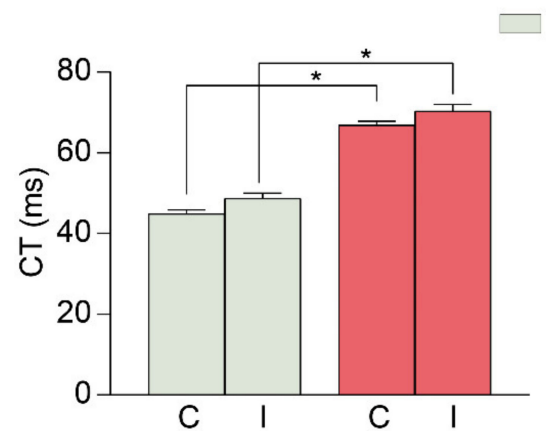

C

NeuroHeal treatment
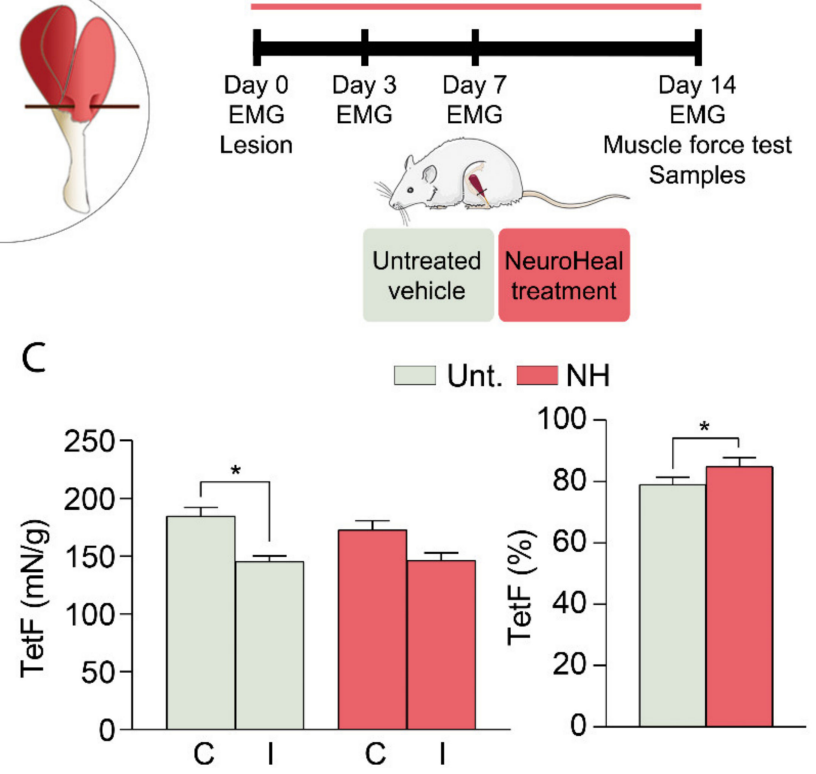

$\square$ Unt. $\square \mathrm{NH}$

$\mathrm{E}$

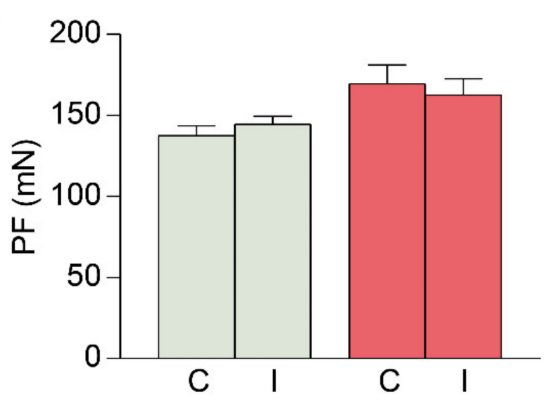

Figure 1. NeuroHeal-treated animals show a better functional recovery after muscle injury. (A) Schematic workflow and experimental groups. (B) Percentage of the compound muscle action potential (CMAP) amplitude of the ipsilateral gastrocnemius (GA) muscle with respect to the contralateral one from injured untreated (Unt., $n=13$ ) and injured treated with NeuroHeal (NH, $n=11$ ) groups (two-way ANOVA, ${ }^{*} p<0.05,{ }^{* *} p<0.01, * * * p<0.001$ vs. 0 dpi for each respective group; \# $p<0.05$, \#\# $p<0.01$ vs. Unt. for each dpi; $\$ p<0.05$ vs. 3 dpi in NH group). (C) Left, bar graph of the average of the maximum tetanus force (TetF) from ipsi- (I) and contralateral (C) GA muscle from Unt. and NH at 14 dpi ( $n=5$; one-way ANOVA, ${ }^{*} p<0.01$ ). Right, bar graph of the percentage of TetF of the ipsilateral GA muscle respect to the contralateral one from Unt. and NH at 14 dpi $(n=5 ; t$-test * $p=0.05)$. (D) Left, bar graph of the average of the contraction time (CT) from ipsi- (I) and contralateral (C) GA muscle from GA from Unt. and NH at 14 dpi $\left(n=5\right.$; one-way ANOVA, $\left.{ }^{*} p<0.0001\right)$. Right, bar graph of the average of the half-relaxion time (HRT) from ipsi- (I) and contralateral (C) GA muscle from Unt. and NH at 14 dpi $\left(n=5\right.$; one-way ANOVA, $\left.{ }^{*} p<0.0001\right)$. (E) Bar graph of the average of the peak force (PF) from ipsi- (I) and contralateral (C) GA muscle from GA from Unt. and NH at 14 dpi ( $n=5$; one-way ANOVA). 


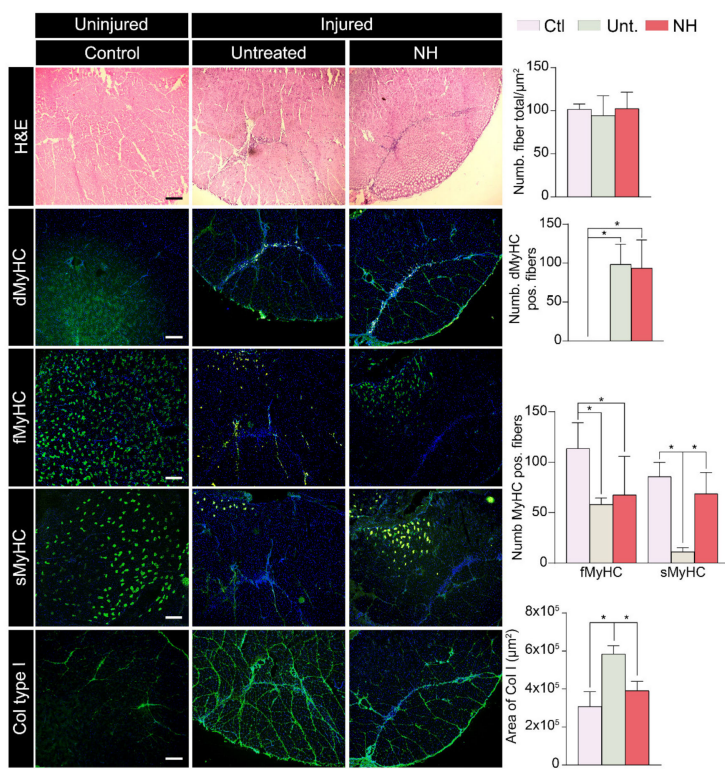

Figure 2. NeuroHeal reduces collagen deposition and promotes slow-twitch fiber typing after muscle injury. Left, representative microphotographs of contralateral control healthy gastrocnemius (GA) muscle sections (Ctl) and lesioned GA muscle sections from the injured untreated (Unt.) and injured treated with NeuroHeal $(\mathrm{NH})$ groups at $14 \mathrm{dpi}$. Scale bar $500 \mu \mathrm{m}$, for all corresponding microphotographs as represented in the first image panel, the control condition. First panel, left, representative microphotographs of GA muscle sections stained with H\&E. Right, bar graph of the average number of muscle fibers ( $n=4$; $t$-test). Second panel, left, representative microphotographs of GA muscle sections immunostained for developmental MyHC. Right, bar graph of the average number of positive fibers for $\mathrm{dMyHC}$ related to the total area of the image $(n=4 ; t$-test). Third and fourth panels, left, representative microphotographs of GA muscle sections immunostained for fast and slow MyHC. Right, bar graph of the average number of positive fibers for $\mathrm{fMyHC}$ and sMyHC related to the total area of the image $\left(n=3 ; t\right.$-test, $\left.{ }^{*} p<0.01\right)$. Fifth panel, left, representative microphotographs of GA muscle sections immunostained for Collagen type I (Col I). Right, bar graph of the average of the area immunolabeled for $\mathrm{Col} I\left(n=4 ;\right.$-test, $\left.{ }^{*} p<0.05\right)$.

\subsubsection{NeuroHeal Influences Muscle Satellite Cells}

We further investigated if NeuroHeal might influence the response of SCs, which are the natural/endogenous way of muscle to recover after injury by regeneration, as they are able to differentiate into myofibers [21]. At 14 dpi, the number of Pax7-positive cells (a classical marker of activated SCs) was similar in the untreated injured animals compared to uninjured controls, while NeuroHeal treatment significantly reduced the quantity (Figure 3). In contrast, there was a significant increase in the number of MyoD(myoblast marker) and MyoG-positive cells (Myogenin, myocyte marker) in injured animals regardless of the treatment, as expected, which suggests commitment of SCs in the myogenic process (Figure 3). 

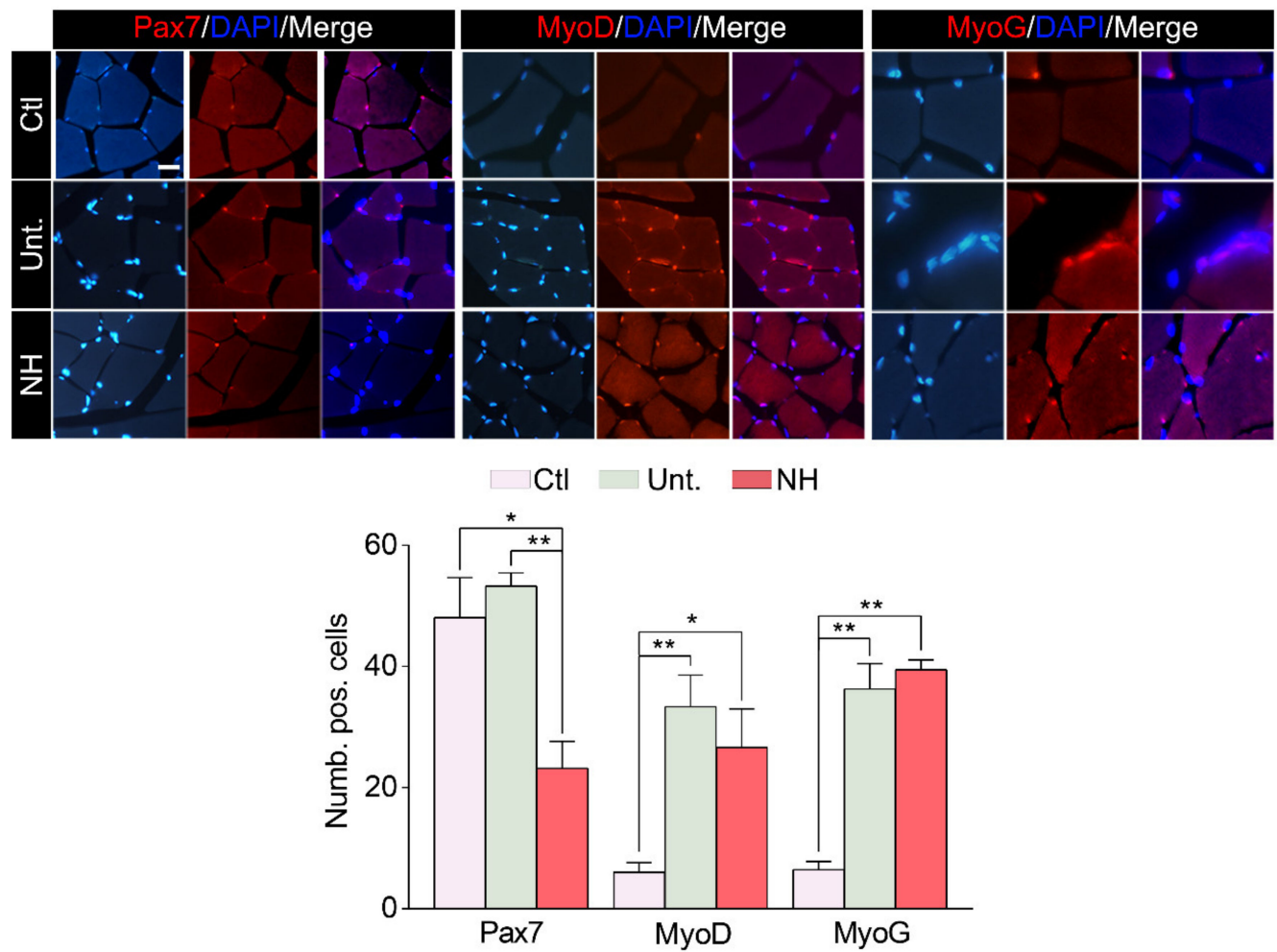

Figure 3. NeuroHeal accelerates satellite cell activation and differentiation after muscle injury. Top, representative microphotographs of the ipsi- and contralateral lesioned gastrocnemius muscle sections revealing the presence of Pax 7, MyoD, and MyoG and stained with DAPI (blue) from the different experimental groups: control (Ctl), injured untreated (Unt.), injured treated with NeuroHeal (NH) at 14 dpi. Scale bar $200 \mu \mathrm{m}$ and identical for all corresponding microphotographs as represented in the first image panel, the control condition. Bottom, bar graphs of the average number of positive satellite nuclei for Pax7, MyoD, or Myogenin $\left(n=3\right.$; one-way ANOVA, $\left.{ }^{*} p<0.05,{ }^{* *} p<0.01\right)$.

We additionally analyzed the differentiation process of myoblasts in vitro to verify the implication of NeuroHeal in this process. The differentiation process of the myoblast cell line C2C12 occurs during 3-5 days of low-serum culture medium [22]. The non-activated and non-quiescent SCs are positive for Ki-67 and negative for Pax7. The following day after the initiation of the differentiation process, the number of $\mathrm{Ki}-67$ positive cells was similar to the non-differentiated cells, but it drastically dropped after 3 div culture (Figure S4B). The use of Ex-527, a selective inhibitor of SIRT1, increased the number of Ki-67 positive and Pax7 positive cells, while the total number of cells was similar to the control (Figure S4C), suggesting the arresting of the differentiation process at this stage in a proportion of cells (Figure $4 \mathrm{~A}$ ). The relative intensity of MyoD within the cells treated with Ex-527 was similar to the control in agreement with normal low activity of SIRT1 at this stage. Treatment with NeuroHeal did not affect the number of Ki-67 positive cells but significantly diminished the number of Pax7 positive cells with respect to the control, suggesting that more cells entered into later stages of differentiation (Figure 4A). Curiously, the number of MyoD positive cells was not affected by NeuroHeal treatment despite the lower intensity of its expression compared to the control (Figure 4A). This observation is consistent with the fact that SIRT1 activation reduces the expression of MyoD [23]. However, the coefficient of fusion of the formed mature myotubes was increased with NeuroHeal compared to the untreated condition, suggesting an acceleration of this differentiation process (Figure 4B). All these effects produced by NeuroHeal were not observed if Ex-527 was concomitantly added to the wells, suggesting that the activation of SIRT1 might be involved, although other factors may play a role and compensating some effects. Finally, we analyzed parvalbumin levels in vivo and in vitro to verify the interaction between SIRT1 and parvalbumin in the differentiation process [24]. NeuroHeal treatment showed a reduction in the relative 
intensity of parvalbumin on myotubes of C2C12 cells (Figure S5A) and also in the in vivo analysis in the intact contralateral GA muscle (Figure S4B). This reduction was reverted by the addition of Ex-527 (Figure S5A). Altogether, these results suggest that NeuroHeal modulates the differentiation process of the myoblast $\mathrm{C} 2 \mathrm{C} 12$ cell line in vitro, increasing the fusion of the new formed matured myotubes, and that SIRT1 activation is involved on it.

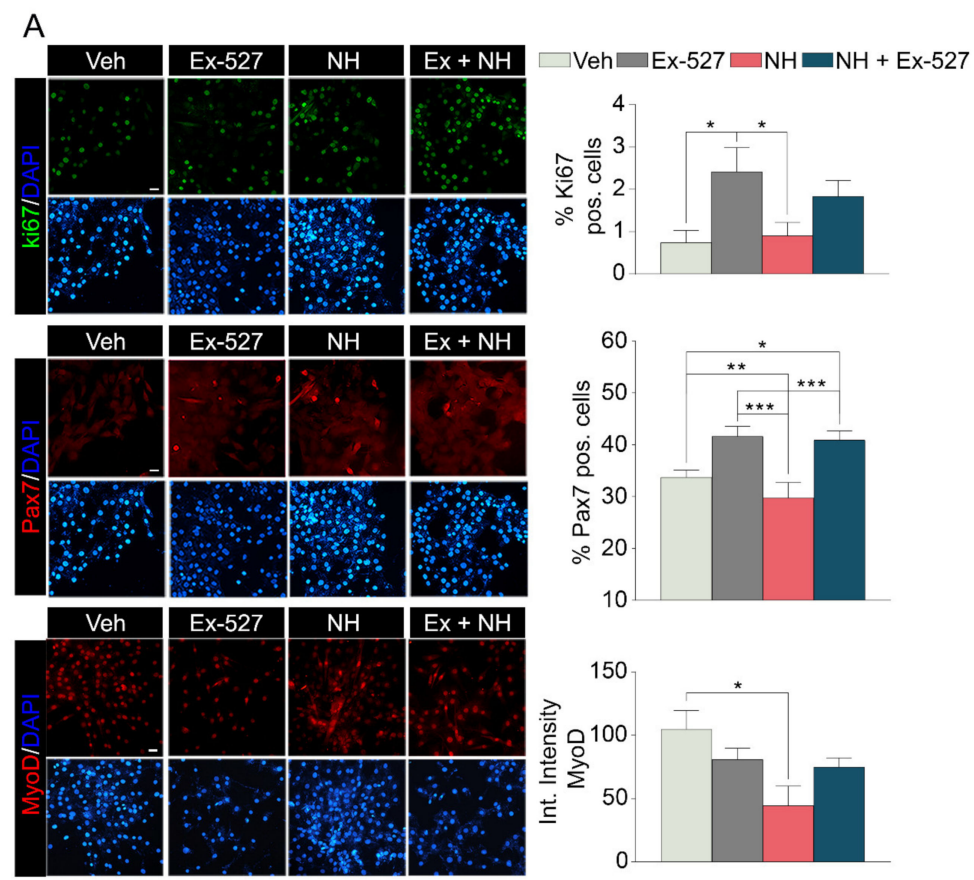

B

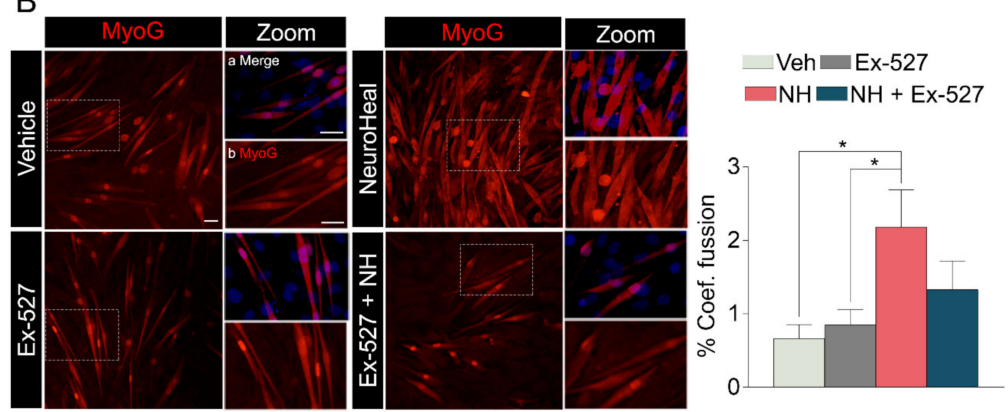

Figure 4. NeuroHeal modulates differentiation process and increases myoblast fusion in vitro. (A) Left, representative microphotographs of differentiated $\mathrm{C} 2 \mathrm{C} 12$ myoblast cell line immunostained for Ki67, Pax7, and MyoD and stained with DAPI from the different experimental groups at 3 days of differentiation: atrophy-induced treated with vehicle (Veh), atrophy-induced treated with Ex-527 (Ex-527), atrophy-induced treated with NeuroHeal (NH), and atrophy-induced treated with $\mathrm{NH}$ plus Ex-527. Scale bar $25 \mu \mathrm{m}$ and identical for all corresponding microphotographs as represented in the first image panel, the vehicle condition. Right, bar graph of the average number of positive nuclei for Ki67 and Pax7, and the relative intensity per cell of MyoD ( $n=3$; one-way ANOVA, ${ }^{*} p<0.05,{ }^{* *} p<0.01,{ }^{* * *} p<0.001$ ). (B) Left, representative immunofluorescence microphotographs of differentiated C2C12 myoblast cell line immunostained for MyHC and stained with DAPI (blue) from the different experimental groups at 5 days of differentiation. Panels a and $b$ are zoom images for each condition from the images of the left. The scale bar is $200 \mu \mathrm{m}$ for all corresponding microphotographs as represented in the first image panel, the vehicle condition, and zoomed images. Right, bar graph of the percentage of the number of positive myotubes with three or more nuclei $(n=3$, one-way ANOVA, $\left.{ }^{*} p<0.05\right)$. 


\section{Discussion}

The results of this work indicate that NeuroHeal affects muscle cell biology, improving muscle regeneration and accelerating myogenesis after injury. These beneficial effects are accompanied by reduced collagen deposition and an increased number of mature muscle fibers, preferentially of the slow-twitch type. Overall, NeuroHeal may be a promising treatment to improve skeletal muscle regeneration after damage and for some musclerelated diseases.

Although NeuroHeal was discovered to act on the nervous system, in previous studies, we observed that it also reduces muscle atrophy caused by denervation [12] and by hindlimb immobilization [17]. For that reason, we pursued to decipher whether it may act on muscle cell biology as well by using a rat model of sports-type muscle injury [20]. Surprisingly, we observed that the treatment with NeuroHeal advanced the recovery of the electrophysiological response, and promoted a tendency to increase the TetF of the damaged muscle. This was accompanied by observations in the histological analysis; less collagen deposition, which contributes to form scar tissue, and an increasing number of mature muscle fibers, in particular slow-twitch fibers. These observations are in agreement with the known mechanism of action of NeuroHeal as a SIRT1 activator $[18,25]$. Several studies have shown that activation of SIRT1 in muscle disease models promotes shifting from fast to slow-twitch fibers [26-28]. However, we did not observe such an increase in this type of fibers in the uninjured muscles in our model, suggesting that more time of treatment is necessary under normal conditions or that this only occurs when the muscle is affected/lesioned. In any case, it would be worth exploring these possibilities.

In the muscular force analysis, there was an unexpected increase in the contraction and relaxation time produced by NeuroHeal treatment, due to a PF light increase. This may be attributed to an increase in the pulse of free $\mathrm{Ca}^{+2}$ in the cytoplasm. Two possible mechanisms may contribute to this action. First, it is reported that one action of NeuroHeal's compound ACA is the activation of the VGCC channel [29], which provides $\mathrm{Ca}^{+2}$ entry activating the RyR. This prolongation of the RyR activation could explain the increase in the $\mathrm{CT}$ parameter. A second reason may be related to the reduction of parvalbumin produced by NeuroHeal, which acts as a $\mathrm{Ca}^{+2}$ binding protein, extending the concentration of free $\mathrm{Ca}^{+2}$. This would impede the muscle to relax, leading to an HRT increase. In addition, these low levels are in agreement with findings reported by Ducreux and collaborators [24]. They reported that parvalbumin expression and mitochondrial volume in muscle cells are inversely regulated via a SIRT1/PGC- $1 \alpha$ signaling axis. We did not explore the action of NeuroHeal on mitochondria biogenesis, but considering the importance of parvalbumin in buffering $\mathrm{Ca}^{+2}$ during the relaxation/contraction process, this may explain why the times for contraction and relaxation were higher in the animals treated with NeuroHeal compared to controls in both contralateral and ipsilateral muscles.

Finally, we explored the effects of NeuroHeal on SCs, which normally remain in a quiescent state until they receive activating signals to begin proliferation $[30,31]$. SC activation is metabolically highly demanding, and it is associated with a large increase in cellular ATP. Indeed, it was found that SIRT1, a key nutrient sensor, modulates autophagic flux during SC activation [32]. The activation of the autophagic machinery by SIRT1 is necessary in order to generate nutrients, which are essential for the generation of ATP, to support the considerable increase in anabolic activity associated with the activation process. Inhibition of SIRT1 or autophagy causes a delay in SC activation [33]. We have previously found that NeuroHeal activates SIRT1 and favors the autophagy flux [14], so these may be causing either the effect of a quick activation of myoblast observed in vitro or the diminished number of Pax7 positive SC in vivo compared to the untreated animals. The same number of Pax7 positive SC in vivo in the untreated and control groups could be caused by a delayed activation of the SC in contrast to an early induction due to SIRT1 activation. Upon SC activation, the metabolic switch from fatty acid oxidation to glycolysis produces a drop in free NAD+, and SIRT1 loses its catalytic activity, which allows the expression of muscle-specific genes, such as MyoD [34]. We observed that NeuroHeal 
treatment did not prevent the ability of cells to finish myogenesis and even reduced MyoD expression, which is in agreement with what is reported on SIRT1 action [34]. Furthermore, we observed an increase in the fusion coefficient to form myotubes with NeuroHeal treatment, which, again, could be related to the activation of SIRT1 and its favorable action in the respiration of mitochondria [35].

Overall, we conclude that NeuroHeal could be used clinically to accelerate muscle regeneration, and probably also as a treatment in various muscle diseases due to its ability to activate SIRT1 [36].

Supplementary Materials: The following are available online at https://www.mdpi.com/2073-4 409/10/1/22/s1, Figure S1: Muscle injury does not modify muscle weight. Figure S2: NeuroHeal reduces collagen deposition and promotes slow-twitch fiber typing after muscle injury. Figure S3: NeuroHeal does not modify the myosin-fiber pattern in healthy muscle. Figure S4: C2C12 cell line maintains a high rate of proliferation one day after differentiation. Figure S5: Parvalbumin is modulated by SIRT1 in vivo and in vitro.

Author Contributions: S.M.-M.-A. and D.R.-G. conceived the research, performed the experiments, analyzed the results, and wrote the paper. M.M. and V.V. designed and performed the in vivo muscle injury model and muscle force analysis. C.C. conceived the research and contributed to writing the paper. All the authors approved the final version of the manuscript. All authors have read and agreed to the published version of the manuscript.

Funding: This work was supported by the Ministerio de Economía y Competitividad of Spain [grant number \#SAF 2014-59701] and FEDER funds. We are also grateful for the support of TERCEL and CIBERNED from the Instituto de Salud Carlos III of Spain.

Institutional Review Board Statement: The study was conducted according to the guidelines of the Declaration of Helsinki, and approved by the Institutional Animal Care and Use Committee at Vall d'Hebron Hospital Research Insti-tute (Barcelona, Spain) (2017/52.17).

Informed Consent Statement: Not applicable.

Data Availability Statement: The data presented in this study are available on request from the corresponding author. The data are not publicly available due to patent issues.

Special Statement: The authors wish to dedicate a special mention in memory of Caty Casas, an influential scientist, and a wonderful and brave person, who died prematurely at the age of 52 years. It has been an honor and a privilege to share part of her life, her professional capacity, and her ethical and human values.

Acknowledgments: The 2E-8, A474, BAF8, F1652, 8-3A5, and Pax7 were obtained from the Developmental Studies Hybridoma Bank developed under the auspices of the NICHD and maintained by the University of Iowa, Department of Biology. The authors thank Xavier Navarro for revising the manuscript.

Conflicts of Interest: The authors declare no conflict of interest.

\section{References}

1. Chan, O.; Del Buono, A.; Best, T.M.; Maffulli, N. Acute muscle strain injuries: A proposed new classification system. Knee Surg. Sport. Traumatol. Arthrosc. 2012, 20, 2356-2362. [CrossRef] [PubMed]

2. Huard, J.; Li, Y.; Fu, F.H. Muscle injuries and repair: Current trends in research. J. Bone Jt. Surg.-Am. Vol. 2002, 84, 822-832. [CrossRef]

3. Canata, G.L.; D’Hooghe, P.; Hunt, K.J. Muscle and Tendon Injuries; Canata, G.L., D’Hooghe, P., Hunt, K.J., Eds.; Springer: Berlin/Heidelberg, Germany, 2017; ISBN 978-3-662-54183-8.

4. Starkey, J.D.; Yamamoto, M.; Yamamoto, S.; Goldhamer, D.J. Skeletal Muscle Satellite Cells Are Committed to Myogenesis and Do Not Spontaneously Adopt Nonmyogenic Fates. J. Histochem. Cytochem. 2011, 59, 33-46. [CrossRef]

5. Pallafacchina, G.; Blaauw, B.; Schiaffino, S. Role of satellite cells in muscle growth and maintenance of muscle mass. Nutr. Metab. Cardiovasc. Dis. 2013, 23, S12-S18. [CrossRef]

6. Rocheteau, P.; Vinet, M.; Chretien, F. Dormancy and Quiescence of Skeletal Muscle Stem Cells. In Results and Problems in Cell Differentiation; Springer Verlag: New York, NY, USA, 2015; Volume 56, pp. 215-235.

7. Lepper, C.; Partridge, T.A.; Fan, C.-M. An absolute requirement for Pax7-positive satellite cells in acute injury-induced skeletal muscle regeneration. Development 2011, 138, 3639-3646. [CrossRef] 
8. Sambasivan, R.; Yao, R.; Kissenpfennig, A.; Van Wittenberghe, L.; Paldi, A.; Gayraud-Morel, B.; Guenou, H.; Malissen, B.; Tajbakhsh, S.; Galy, A. Pax7-expressing satellite cells are indispensable for adult skeletal muscle regeneration. Development 2011, 138, 3647-3656. [CrossRef]

9. Grassi, A.; Napoli, F.; Romandini, I.; Samuelsson, K.; Zaffagnini, S.; Candrian, C.; Filardo, G. Is Platelet-Rich Plasma (PRP) Effective in the Treatment of Acute Muscle Injuries? A Systematic Review and Meta-Analysis. Sport. Med. 2018, 48, 971-989. [CrossRef]

10. Contreras-Muñoz, P.; Torrella, J.R.; Serres, X.; Rizo-Roca, D.; De La Varga, M.; Viscor, G.; Martínez-Ibáñez, V.; Peiró, J.L.; Järvinen, T.A.H.H.; Rodas, G.; et al. Postinjury Exercise and Platelet-Rich Plasma Therapies Improve Skeletal Muscle Healing in Rats but Are Not Synergistic When Combined. Am. J. Sports Med. 2017, 45, 2131-2141. [CrossRef]

11. Qazi, T.H.; Duda, G.N.; Ort, M.J.; Perka, C.; Geissler, S.; Winkler, T. Cell therapy to improve regeneration of skeletal muscle injuries. J. Cachexia. Sarcopenia Muscle 2019, 10, 501-516. [CrossRef] [PubMed]

12. Romeo-Guitart, D.; Forés, J.; Navarro, X.; Casas, C. Boosted Regeneration and Reduced Denervated Muscle Atrophy by NeuroHeal in a Pre-clinical Model of Lumbar Root Avulsion with Delayed Reimplantation. Sci. Rep. 2017, 7, 1-12. [CrossRef] [PubMed]

13. Romeo-Guitart, D.; Casas, C. Network-centric medicine for peripheral nerve injury: Treating the whole to boost endogenous mechanisms of neuroprotection and regeneration. Neural Regen. Res. 2019, 14, 1122. [CrossRef] [PubMed]

14. Romeo-Guitart, D.; Marcos-DeJuana, C.; Marmolejo-Martínez-Artesero, S.; Navarro, X.; Casas, C. Novel neuroprotective therapy with NeuroHeal by autophagy induction for damaged neonatal motoneurons. Theranostics 2020, 10, 5154-5168. [CrossRef] [PubMed]

15. Romeo-Guitart, D.; Leiva-Rodriguez, T.; Forés, J.; Casas, C. Improved Motor Nerve Regeneration by SIRT1/Hif1a-Mediated Autophagy. Cells 2019, 8, 1354. [CrossRef] [PubMed]

16. Romeo-Guitart, D.; Casas, C. NeuroHeal Treatment Alleviates Neuropathic Pain and Enhances Sensory Axon Regeneration. Cells 2020, 9, 808. [CrossRef] [PubMed]

17. Marmolejo-Martínez-Artesero, S.; Romeo-Guitart, D.; Mañas-García, L.; Barreiro, E.; Casas, C. NeuroHeal Reduces Muscle Atrophy and Modulates Associated Autophagy. Cells 2020, 9, 1575. [CrossRef]

18. Romeo-Guitart, D.; Forés, J.; Herrando-Grabulosa, M.; Valls, R.; Leiva-Rodríguez, T.; Galea, E.; González-Pérez, F.; Navarro, X.; Petegnief, V.; Bosch, A.; et al. Neuroprotective Drug for Nerve Trauma Revealed Using Artificial Intelligence. Sci. Rep. 2018, 8, 1-15. [CrossRef] [PubMed]

19. Harriss, D.; Atkinson, G. Ethical Standards in Sport and Exercise Science Research: 2014 Update. Int. J. Sports Med. 2013, 34, 1025-1028. [CrossRef]

20. Contreras-Muñoz, P.; Fernández-Martín, A.; Torrella, R.; Serres, X.; De La Varga, M.; Viscor, G.; Järvinen, T.A.H.; Martínez-Ibáñez, V.; Peiró, J.L.; Rodas, G.; et al. A New Surgical Model of Skeletal Muscle Injuries in Rats Reproduces Human Sports Lesions. Int. J. Sports Med. 2016, 37, 183-190. [CrossRef]

21. Gayraud-Morel, B.; Chrétien, F.; Tajbakhsh, S. Skeletal muscle as a paradigm for regenerative biology and medicine. Regen. Med. 2009, 4, 293-319. [CrossRef]

22. Dugdale, H.F.; Hughes, D.C.; Allan, R.; Deane, C.S.; Coxon, C.R.; Morton, J.P.; Stewart, C.E.; Sharples, A.P. The role of resveratrol on skeletal muscle cell differentiation and myotube hypertrophy during glucose restriction. Mol. Cell. Biochem. 2018, 444, 109-123. [CrossRef]

23. Amat, R.; Planavila, A.; Chen, S.L.; Iglesias, R.; Giralt, M.; Villarroya, F. SIRT1 Controls the Transcription of the Peroxisome Proliferator-activated Receptor- $\gamma$ Co-activator- $1 \alpha$ (PGC- $1 \alpha$ ) Gene in Skeletal Muscle through the PGC-1 $\alpha$ Autoregulatory Loop and Interaction with MyoD. J. Biol. Chem. 2009, 284, 21872-21880. [CrossRef] [PubMed]

24. Ducreux, S.; Gregory, P.; Schwaller, B. Inverse Regulation of the Cytosolic Ca2+ Buffer Parvalbumin and Mitochondrial Volume in Muscle Cells via SIRT1/PGC-1 $\alpha$ Axis. PLoS ONE 2012, 7, e44837. [CrossRef] [PubMed]

25. Romeo-Guitart, D.; Leiva-Rodríguez, T.; Espinosa-Alcantud, M.; Sima, N.; Vaquero, A.; Domínguez- Martín, H.; Ruano, D.; Casas, C.; Domínguez-Martín, H.; Ruano, D.; et al. SIRT1 activation with neuroheal is neuroprotective but SIRT2 inhibition with AK7 is detrimental for disconnected motoneurons. Cell Death Dis. 2018, 9, 531. [CrossRef] [PubMed]

26. Zhang, C.; Luo, J.; Yu, B.; Zheng, P.; Huang, Z.; Mao, X.; He, J.; Yu, J.; Chen, J.; Chen, D. Dietary resveratrol supplementation improves meat quality of finishing pigs through changing muscle fiber characteristics and antioxidative status. Meat Sci. 2015, 102, 15-21. [CrossRef] [PubMed]

27. Lagouge, M.; Argmann, C.; Gerhart-Hines, Z.; Meziane, H.; Lerin, C.; Daussin, F.; Messadeq, N.; Milne, J.; Lambert, P.; Elliott, P.; et al. Resveratrol Improves Mitochondrial Function and Protects against Metabolic Disease by Activating SIRT1 and PGC-1 $\alpha$. Cell 2006, 127, 1109-1122. [CrossRef] [PubMed]

28. Ljubicic, V.; Burt, M.; Lunde, J.A.; Jasmin, B.J. Resveratrol induces expression of the slow, oxidative phenotype in mdx mouse muscle together with enhanced activity of the SIRT1-PGC-1 $\alpha$ axis. Am. J. Physiol. Physiol. 2014, 307, C66-C82. [CrossRef] [PubMed]

29. Feng, Y.; Wang, B.; Du, F.; Li, H.; Wang, S.; Hu, C.; Zhu, C.; Yu, X. The Involvement of PI3K-Mediated and L-VGCC-Gated Transient Ca2+ Influx in 17 $\beta$-Estradiol-Mediated Protection of Retinal Cells from H2O2-Induced Apoptosis with Ca2+ Overload. PLoS ONE 2013, 8, e77218. [CrossRef]

30. Brack, A.S.; Rando, T.A. Tissue-Specific Stem Cells: Lessons from the Skeletal Muscle Satellite Cell. Cell Stem Cell 2012, 10, 504-514. [CrossRef] 
31. Yin, H.; Price, F.; Rudnicki, M.A. Satellite cells and the muscle stem cell niche. Physiol. Rev. 2013, 93, 23-67. [CrossRef]

32. Ng, F.; Tang, B.L. Sirtuins' modulation of autophagy. J. Cell. Physiol. 2013, 228, 2262-2270. [CrossRef]

33. Tang, A.H.; Rando, T.A. Induction of autophagy supports the bioenergetic demands of quiescent muscle stem cell activation. EMBO J. 2014, 33, 2782-2797. [CrossRef] [PubMed]

34. Ryall, J.G.; Orso, S.D.; Derfoul, A.; Juan, A.; Zare, H.; Feng, X.; Clermont, D.; Koulnis, M.; Gutierrez-cruz, G.; Sartorelli, V. The NAD+ -Dependent SIRT1 Deacetylase Translates a Metabolic Switch into Regulatory Epigenetics in Skeletal Muscle Stem Cells. Cell Stem Cell 2016, 16, 171-183. [CrossRef] [PubMed]

35. Wu, Z.; Puigserver, P.; Andersson, U.; Zhang, C.; Adelmant, G.; Mootha, V.; Troy, A.; Cinti, S.; Lowell, B.; Scarpulla, R.C.; et al. Mechanisms Controlling Mitochondrial Biogenesis and Respiration through the Thermogenic Coactivator PGC-1. Cell 1999, 98, 115-124. [CrossRef]

36. Tonkin, J.; Villarroya, F.; Puri, P.L.; Vinciguerra, M. SIRT1 signaling as potential modulator of skeletal muscle diseases. Curr. Opin. Pharmacol. 2012, 12, 372-376. [CrossRef] 